\title{
Asthma, Allergy, Mood Disorders, And Nutrition
}

\author{
A. Wilczynska-Kwiatek, K. Bargiel-Matusiewicz, L. Lapinski \\ Institute of Psychology, University of Silesia, Katowice, Poland
}

\begin{abstract}
Background: Growing evidence supports comorbidity of asthma and allergies with mood disorders and various connections between these diseases. It still remains unclear whether this comorbidity is caused by the same pathophysiological factors or whether there are other links between asthma and depression. There is no definite answer to the question of an optimal treatment to deal with both asthma and depression, when they occur simultaneously. Epidemiological and clinical trials on the influence of nutrition on certain diseases suggest the effects of $\omega 3$ polyunsaturated fatty acids (PUFAs) in aiding treatment of mood disorders and inflammatory conditions.

Objective: This is an overview showing the connections between asthma, allergic disease, and mood disorders, and the influence of nutrition on these conditions. Evidence indicates positive correlations between consumption of PUFAs and mood correction. Several analyses show the connection between diet and asthma. They may form a basis for potential recommending 13 PUFAs as an adjuvant in prevention and treatment of mental disorders, asthma, and allergy.
\end{abstract}

Key words: asthma, allergy, mood disorders, depression, polyunsaturated fatty acids

\section{INTRODUCTION}

Mood disorders, including major depressive disorder and bipolar disorder are recurrent and potentially lifethreatening illnesses. According to the World Health Organisation, depression is one of the most prominent diseases worldwide, a fact reflected in a huge increase in the prescription of anti-depressive drugs in Western World, now about three times as many as just two decades ago. The lifetime prevalence of depression in modern society is estimated between 5 and 11 $\%$ of the population [1]. Depression is the most frequent psychiatric disorder and usually affects well-being in a more fundamental way than other disorders. What is more, a majority of those who commit suicide are depressed at the time of taking one's life. Depression is certainly important risk factor for suicide.

The problem of bronchial asthma affects approximately $10 \%$ of the population in the Western World [2] and is the most common chronic disease among children, $20-25 \%$ of whom experience some sort of bronchial wheezing at least once in their lives. In Europe, the proportion of persons under 14 who suffer from asthma varies from 2 to $20 \%$ of the population.

Allergic disease is a common illness, affecting about $50 \%$ of the population in different forms [3]. Manifestations of allergy are usually debilitating, very bothersome, and in some situations might be life-threatening. In the lungs, allergy may manifest by bronchoconstriction or asthma, in the nose - by rhinitis, in the skin - by urticaria; and in the eyes, by conjunctivitis. The most common form is allergic rhinitis in both perennial and seasonal type due to the increased allergen exposure (e.g., house dust mite or seasonal grass, tree and grain pollens, etc.) This condition is believed to affect between 10 and $30 \%$ of the population [4], which exceed the prevalence of major depression by at least two-fold.

The distinction between nasal inflammation and asthma may be blurred since some authors assume that more than $60 \%$ of patients with allergic rhinitis also have asthma, and rhinitis often develops into allergic asthma. A number of investigations suggest that allergies and asthma are linked to stress and various psychological disorders and dysfunctions, particularly to anxiety and depression. However, it cannot be definitely stated whether depression is a risk factor of asthma or vice versa. What has been determined as a fact is that the two have comorbidity and are probably correlated in a significant way.

In the case of some diseases, including those relating to mental health and asthma, various studies are conducted to verify the hypothesis about the dietary influence on health, and particularly the role of polyunsaturated fatty acids (PUFAs).

\section{Material AND Methods}

In this article we present an overview of the examined connections between asthma, allergic disorder, and mood disorders, and we outline studies verifying the influence of nutrition on mood disorders. To accomplish this goal, PubMed, EBSCO and other databases were searched for all years of records until February 2009. Relevant published studies are considered and shortly discussed.

\section{RESULTS}

\section{Relationship Between MoOd Disorders, Asthma,} AND AlLERgic Diseases

Asthma is considered as a prototypical chronic physical condition which has been related to anxiety and mood 
disorders. Asthma is one of most common chronic respiratory system disease. It manifests with recurring, variable symptoms such as airflow obstruction, bronchial hyperresponsiveness with underlying inflammatory state. A considerable number of studies examined the interaction between anxiety, depression, suicidal tendencies, allergy and asthma. Mood disorders and asthma are common comorbid diseases with possibly shared pathophysiology, influenced among others by chosen treatment and various personality constructs (i.e., neuroticism, locus of control). Similar relations are being indicated in terms of mood disorders and allergy. Still, many studies are ongoing to further investigate this association. Here, we would like to point out several identified connections between these conditions.

It has been repeatedly reported that allergic rhinitis and asthma are associated with higher rates of depression and anxiety disorder. Observation and clinical trials often confirm that asthmatics have significantly higher results for anxiety and depression than the general population. Zielinski et al. [5] reviewed eight studies on the prevalence of depressive symptoms in children and adults suffering from asthma. All of them indicated that depressive symptoms occur with significantly greater frequency among asthmatics than in the general population.

Epidemiological survey conducted by Cooper et al. [6] indicates that approximately $1 / 3$ of asthmatics experience anxiety at a level approaching clinical disorder, which is double that in general population. They have also observed that the strength of the relation between asthma and psychological stress depends on age and sex. The prevalence of anxiety is higher in women then in men with asthma and increases with age. Interestingly, authors reported that asthma is related rather with generalized anxiety than asthma specific anxiety. Thus, it is likely that anxiety experienced in asthma is a part of a wider picture of global psychological distress rather than indicating that panic fear has a specific role in this disease.

For many years, psychological distress has been believed to have a significant meaning in asthma, since asthmatics tend to have higher levels of stress and negative mood states (fear, irritability, depression, etc.). Evidence shows that the level of stress and sense of control in persons with asthma may be related to a significant degree on the intensity of disease symptoms [7]. Persons suffering simultaneously from asthma and anxiety or depressive disorders often experience more frequent asthma symptoms than those without psychological conditions. In fact, emotional strain can exacerbate the physical manifestations in atopic disorders and even might function as a kind of trigger in asthma attacks. Liu et al. [8] performed an antigen challenge on students during both low stress (during mid-semester) and high stress period (during final examination). The authors implied that psychological stress can act as a cofactor to increase eosinophilic airway inflammatory responses to antigen challenge and increases asthma severity. It is, therefore, worth considering the recommendation of routine stress-management for asthma sufferers.

As far as allergic disease is concerned, Goodwin et al. [9] have proposed a hypothesis that the presence of specific personality factors in large measure can explain the connection between allergy and depression. The authors focused on neuroticism. In their opinion, higher level of neuroticism is connected with a higher risk of occurrence of allergies, and the strength of the relationship between allergy and depression is likely to be moderated by neuroticism.

Patients might find it difficult to differentiate between anxiety symptoms (e.g., panic fear attacks) and those of asthma (cough, difficulties with breathing) because they may appear quite similar. For that reason, many of those who suffer from both may seek treatment for only one (e.g., for asthma) and miss the other. It is also possible that some of them may not be fully diagnosed. Such situation could lead to inappropriate medication. A research on management errors in severe asthma in adults showed that most of them were related to patient behavior and that panic fear and overuse of beta-agonists were important contributory factors.

Very often asthma is associated with sleep abnormalities. A survey study of over 7500 participants affected with asthma reported that $74 \%$ of them have woken up in the middle of the night once a week, because of severe asthma symptoms (cough, breathing problems). In the same survey $40 \%$ of participants confirmed that this problem occurs every night [10].

Insufficient rest at night might lead to chronic fatigue feeling during the day, attention depletion, decreased efficacy at work, depressed mood, etc. It may be assumed that sleep disturbances caused by asthma or allergy may bring about or exacerbate depressive symptoms. Functional impairment due to lack of sleep is often associated with gradual withdrawal in physical and social activity, which increases affective symptoms in a feedback manner.

Depression, anxiety and sleep disturbances are key suicide risk factors. As their rates are greater in allergic patients than in general population, there have been studies conducted concerning the relationship between allergy and suicide risk. It might be suggested that people with a history of allergy have an increased suicide tendencies and higher suicide rate than those without allergy. A great number of epidemiologic studies confirmed that every year, the highest levels in suicide acts and the peak in depressive episodes are noted in spring. Postolache et al. [11] suggest that time convergence of these peaks is related with seasonal allergen exposure. In example, grass and tree pollen has their peak pollen season in spring. Investigators found that the relative rate of suicide in young women $(\mathrm{IgE}$ anti-tree pollen positive) increased two-fold during the peak of tree pollen season as compared with low tree pollen season. Moreover, certain pharmaceuticals used in allergy treatment potentially can exacerbate suicide risk (i.e., systemic corticosteroids or montelukast, due to increasing depressive symptoms). However, further trials in this area seem necessary.

\section{OMega-3 And Omega-6 Polyunsaturated FatTy ACIDS}

The $\omega 3$ and $\omega 6$ polyunsaturated fatty acids are essential fatty acids in many mammals, including humans, as 
they cannot be synthesized by organism and they have to be obtained from the diet. The $\omega 3$ PUFAs are present in linseed oil, walnuts, wheat, soya beans, and particularly in cold water sea fish (like tuna, salmon, herring, etc), while maize, sunflower and sesame oil are main sources of $\omega-6$ PUFAs.

There is biochemical data that n-3 PUFAs play an important role in neural structure and function. The brain and central nervous system (CNS) contain high concentration of $\omega-3$ PUFAs and several studies suggest a role for n-3 PUFAs in neurotransmitter synthesis, degradation, release, reuptake, and binding. Fatty acids constitute part of phospholipids and, consequently, of all biological membranes. The membrane fluidity, of crucial importance for its functioning, depends on its lipid components. A diet rich in essential polyunsaturated fatty acids allows a higher incorporation of cholesterol in the membranes to balance their fluidity,

The significant factor in fatty acids efficacy does not seem to be their absolute level, but the ratio between various groups of fatty acids. It is known that the relative amounts of $\omega 6$ and $\omega 3$ PUFAs in the cell membrane are responsible for affecting cellular function. Some authors indicate the optimal ratios of polyunsaturated:saturated $=\omega 6: \omega 3=1: 1$.

The types of fatty acids that are available to the composition of cell membranes depend upon diet. Retina and brain, particularly cerebral cortex, are rich in omega- 3 fatty acids and the role of $n-3$ in visual and cognitive development has been widely examined. Any dietary deficits of essential PUFAs have consequences on cerebral development, modifying the activity of enzymes of the cerebral membranes.

PUFAs are precursors of eicosanoids - prostaglandins and leukotriene, which is involved in inflammation and immune response, a diet rich in fish oil reduces production of PGE2. What is more, an increase in eicosapentenoic acid (EPA) intake leads to a reduction in the production of inflammatory cytokines. So, it might be important to use $\omega 3$ PUFAs in treatment of chronic inflammatory diseases, such as asthma. Finally, it seems that $\omega 3$ PUFAs might prevent the onset of hormone-dependent tumors by antagonizing the formation of cancerogenic factors.

\section{Nutrients, Asthma, AND Allergic Disorder}

The association between polyunsaturated long chain fatty acids, atopy, and asthma has been discussed for decades. In recent years, the effect of diet on various aspects of atopic diseases and asthma has been a subject of increasing number of studies. Gradually, mutual connections seem to become more and more apparent. For instance, there are studies which imply that consumption of certain foods such as milk, apples, and pears reduces the risk of asthma, while soybean products ingestion, however, raises that risk. Also diet rich in polyphenols (particularly flavonoids) is thought to have beneficial influence in asthma prophylactics.

Hitherto, a number of studies have confirmed the role of fatty acids intake in inflammation and autoimmune diseases. Among the fatty acid, n-3 PUFAs seem to have the most important immunomodulatory influ- ence (in particular EPA and DHA). Clinical intervention trials and animal studies report that n-3 PUFAs reveal anti-inflammatory properties and, for that reason, it might become useful in management of inflammatory and autoimmune diseases. There have also been conducted a number of investigations on the role of dietary supplementation with fish oils in asthma and atopic disorders.

The researchers reported statistically significant results indicating a relationship between lower incidence of asthma and allergies and consumption of $\omega 3$ fatty acids. For instance, $\mathrm{Yu}$ and Bjorksten [12] observed that in children $(n=22)$ suffering from asthma or atopic eczema a lower DHA and total PUFA level in the blood and a higher n-6:n-3 ratio than in the control group of healthy children $(n=23)$.

There is also much evidence indicating a beneficial role of fish oil/ $\omega 3$ PUFAs supplementation during pregnancy in modifying allergen-specific immune responses and clinical outcomes in children. As an example, the Swedish study conducted by Duchén et al. [13] compared the chemical composition of breast milk from mothers of children with atopic symptoms and of those without. They have investigated the milk for the composition of PUFAs and secretory immunoglobulin A (S-IgA) levels to nutritional proteins and inhalant allergen (cat). A significantly lower level of EPA, DPA, and DHA was found in the milk of mothers whose children developed allergies than in that of mothers of non-allergic children. In atopic children, a lower PUFA level in phospholipids in the blood was found. Duchen et al. [13] indicate that a low level of $\omega 3$ PUFAs in maternal milk and in particular high arachidonic acid (AA):EPA proportion in blood phospholipids in infants are significantly tied to the development of allergic symptoms in children at 18 months. Interestingly, authors conclude that low $\alpha$ linolenic acid (ALA) and long-chain fatty acid (EPA and DHA) levels have greater significance in the development of atopic disorders in infants than levels of S-IgA antibodies to allergens in the maternal milk. Unfortunately, it is unclear how long this effect lasts. Furthermore, Swedish researchers indicate that fatty acid composition of human milk is dependent on the time of lactation not only during breast meal but also the time of the day and period of lactation.

There is also evidence suggesting that asthma might be ingrained in fetal life and that the intake of n-3 PUFAs during pregnancy might have immunomodulatory effects on the child. Olsen et al. [14] investigated whether $\omega 3$ PUFAs supplementation in pregnancy may affect the child's asthma risk. A population-based sample of over 500 women around pregnancy week 30 was randomly assigned to receive either n-3 PUFAs or placebo capsules until delivery. In a 16 r follow-up authors reported that the hazard rate of asthma was reduced by $63 \%$, while the hazard rate of allergic asthma was decreased by $87 \%$ in the fish oil compared with placebo group. Thus, it might be possible that increasing n-3 PUFAs intake in late gestation may play an important prophylactic role in relation to child's asthma.

The study conducted by Broughton et al. [15] suggests that increasing fatty acids intake can have an effect on asthma symptoms. They have studied the in- 
fluence of $\omega 3$ PUFAs at the ratios of $n-6: n-3$ 10:1 to 5:1 among asthmatics in ameliorating methacholine-induced respiratory stress. Low n-3 PUFA intake was associated with an increase in methacholine-induced respiratory distress. With high 133 PUFAs ingestion, changes in urinary 5 -series leukotriene excretion predicted treatment efficacy in more than $40 \%$ of the responders, while the non-responders had a further loss in respiratory capacity.

\section{Nutrients AND MOOD Disorders}

Long chain polyunsaturated fatty acids are implicated in the development of several psychological conditions and diseases, including dementia, schizophrenia, and depression. $\omega 3$ treatment in these diseases often shows positive effects Description of the effects on depression, although it might be considered as controversial, has led to the conclusion that 13 PUFAs can affect affective function, and may even act as a kind of 'mood stabilizer'. Several lines of evidence suggest that there is a relation in humans between dietary intake of $\omega 3$ PUFAs and depressed mood and anxiety. Diet has been suggested to play a role in depression and it has been widely discussed that the rising rates of this disorder are at least partly due to decreased consumption of $\omega-3$ PUFAs, which are being replaced in the western diet by $n-6$ fatty acids.

There is epidemiological evidence that $\omega 3$ PUFAs play role in affective function. Numerous studies have shown a straight link between the drop in $\omega 3$ PUFAs consumption (sea fish) and the risk of depression, particularly as the incidence of this disorder varies, depending on the country, parallel with fish consumption. In this regard, considerable negative correlations have been demonstrated between worldwide fish consumption and rates of depression. One of the most famous and the most spectacular studies in this area were conducted by Hibbeln [16]. The author showed a strong negative association $(\mathrm{r}=-0.84)$ between fish intake and depression across 13 countries.

Here, it is worth emphasizing that the above results should be treated cautiously, as the majority comes from cross-sectional studies and significant correlations obtained might express differences associated with other uncontrolled factors (i.e., smoking).

A relationship between $\omega 3$ intake and depressed mood has also been found in clinical studies. However, only a few studies have focused on dietary factors, showing their underestimated value in research on depression. Lower concentrations of $\omega 3$ have been repeatedly reported in the plasma/red blood cell membranes in persons with major depressive disorder compared with non-depressed participants.

A role on $\omega 3$ PUFAs supplementation in chronic fatigue syndrome has extensively been investigated at the University of Glasgow [17]. A group of 63 adults with the diagnosis of this disease was enrolled in a double blind placebo controlled trial of essential fatty acid therapy involving daily administration of small doses of $\omega 3$ PUFAs over a three-month period. At 1 month, $74 \%$ of patients on active treatment and 23\% of those on placebo assessed themselves as improved over baseline. At 3 months, the corresponding figures were $85 \%$ and $17 \%$, which means that the placebo group had reverted toward the baseline state while those in the 13 PUFAs group showed continued improvement.

A few studies reported improvements in depressive symptoms associated with bipolar disorder after supplementation with n-3 PUFAs compared with placebo. Stoll et al. [18] observed that, compared to subjects receiving placebo, patients with bipolar disorder who had received fish oils $(6.2 \mathrm{~g} / \mathrm{d}$ EPA and $3.4 \mathrm{~g} / \mathrm{d}$ DHA $)$ for at least 30 days were significantly less likely to relapse and had significantly longer time of remission. It has been reported that $\omega 3$ PUFAs group had a better response than olive oil in depressive symptoms and overall pathology, but not in manic symptoms.

An apparent antidepressant effect of EPA and DHA in treatment of affective disorders has been reported by $\mathrm{Su}$ et al. [19]. Investigators conducted an eight-week double-blind, placebo-controlled trial comparing $\omega-3$ PUFAs $(440 \mathrm{mg} / \mathrm{d}$ EPA + $220 \mathrm{mg} / \mathrm{d}$ DHA) with placebo on top of the usual treatment of patients with major depressive disorder. Interestingly, participants in the EPA + DHA group had significant differences in depression scales (compared with placebo group) from the fourth week of supplementation (and the differences between placebo and $\omega 3$ groups kept growing up until week 8).

Efficacy of $\omega 3$ fatty acids in patients with recurrent self-harm have been assessed in investigation conducted by Hallahan et al. [20]. Patients after an act of repeated self-harm were administrated with either 1.2 $\mathrm{g} / \mathrm{d}$ eicosapentenoic acid plus $0.9 \mathrm{~g} / \mathrm{d}$ docosahexenoic acid or placebo for 12 weeks in addition to standard psychiatric treatment. After supplementation, the $\omega 3$ group, compared with placebo reported significantly greater improvements in suicidality, depression, and daily stresses.

Interesting results on $\omega 3$ PUFAs' effects on mood, cognitive and physiological function have been reported by researchers from the University of Siena in Italy [21].The study aimed to examine the effects of $\omega 3$ supplementation in healthy volunteers performing a series of attention tests. 35-day supplementation with $\omega 3$ PUFAs was associated with clear variations in the profile mood state. The mood analysis showed an increase of vigor and decrease in such mood states as: anger, anxiety, fatigue, depression, and confusion. The reaction time in attention tests decreased only after $\omega 3$. Though it was a relatively small sample, the results seem to be a strong suggestion of beneficial influence of 13 PUFA supplementation on general psychological state, including mood and cognitive function, nonspecific for depressive disorders. Data supplied by Fontani et al. [221] may reinforce the hypothesis of the direct action of $\omega 3$ fatty acids on the central nervous system. Authors claim that the importance of these results have to be considered as strengthened by the fact that they occur within subjects in good health and leading a physically active life in whom $\omega 3$ PUFAs improved an already good condition of well-being.

Though many trials confirm a beneficial role of $\omega 3$ in emotional function, we have to admit that available knowledge in this area is still insufficient. In order to statistically compile and compare data from available 
trials, which differ from each other in methodology, research group selection, dose size, etc., Appleton et al. [22] conducted a study aimed at systematically reviewing published randomized controlled trials investigating the effects of $\omega 3$ PUFAs on depressed mood. Medical and health databases were thoroughly searched for trials that exposed participants to $\omega 3$ PUFAs or fish and measured depressed mood. Their investigation indicates that available studies in this area make it difficult to unambiguously assess the influence of $\omega 3$ PUFAs on emotional function. Trial evidence is still limited and difficult to summarize and evaluate due to considerable heterogeneity. The trials vary widely, not only in terms of approach or quality of trial design but also in terms of the group tested, sample size, composition and quantity of PUFAs taken, length of supplementation period, and psychometric instruments used. Larger trials with adequate capability to detect clinically important benefits are still required.

\section{DisCUSSION}

Increasing prevalence of asthma, atopic diseases and mood disorders in past twenty years reveals a growing need of investigating successive connections between these conditions in order to provide more and more effective prophylactics and therapy. Noteworthy is the fact that in the same period, long chain polyunsaturated fatty acids consumption in western countries decreased dramatically.

In this work we have examined several line of evidence that particular nutrients may influence the structure, biochemistry and function of the central nervous system. A number of epidemiological and clinical studies indicate that there is a potential association between dietary $\omega-3$ intake, mental function and allergic diseases such as asthma.

Over the last decades the typical age for the onset of major depression has declined and its incidence, on the whole, has increased in the Western societies up to twenty-fold since the 1940s. This growth in mood disorders' prevalence cannot be completely explained by better diagnostic criteria or changes in the attitudes of health specialists. Based upon the evidence from epidemiological data, case-control studies, and PUFAs levels in human tissues and their antidepressant effects in clinical trials, PUFAs have shed a light which could help discover the unsolved mystery of depression and connect the mind and body. The deficit of essential fatty acids has been reported to be associated with neurological, cardiovascular, cerebrovascular, autoimmune, inflammatory and cancer diseases, but also with psychological conditions and disorders. It has been suggested that the use of foods rich in $\omega 3$ PUFAs can have a stabilizing effect on mood - through a decrease in negative affective states, such as fear, anger, depression, and probably also by reducing asthma and allergy symptoms. Much evidence indicates that nutrients are rather short-term acting agents, so that in order to maintain potential positive results, it is necessary to take them with a high degree of regularity.

$\omega-3$ fatty acids are often employed in addition to conventional medicine. That is because some psychiatric patients with persistent anxiety and/or depression are dissatisfied with the apparent ineffectiveness of traditional treatments and seek a more holistic approach with fewer side effects. Nevertheless, the role of n-3 PUFAs as an adjunct to antipsychotics and melatonin as a treatment or prophylactic agent for side effects may be considered as ambiguous, requiring further trials with sound methodology [23].

In terms of associations between allergic disease, asthma and nutrition we can say that there is not yet enough evidence in the form of professionally conducted studies. In fact, we do not possess a great deal of research available concerning the connection between $\omega$-3 fatty acids, allergy, and asthma. Nonetheless, it must be emphasized that in spite of the deficiency of wide investigation in the sphere of $\omega 3$ PUFAs dietary intake and asthma, several studies made up to this point indicate the benevolent effects of $\omega-3$ in asthma prevention and treatment. For a reduction in the size of the constantly growing population suffering from asthma is paramount, further investigations in this field are required.

The results mentioned above, force to thorough consideration on the issue of dietary management in asthma treatment and associated disorders such as depression. They provide topics for discussion and further research, which seems crucial. Indeed, the role of $\omega 3$ PUFAs in immunity and affective function may support the promising psychoneuroimmunological hypothesis of depression and provides a great interface of body and mind. It seems that polyunsaturated fatty acids may become useful in prophylactics and/or therapy of allergy and mood disorders in both comorbid and isolated form.

We shall be aware that data concerning the influence of $\omega-3$ PUFAs on mental function, atopic diseases and asthma are relatively limited and often ambiguous. We find it exceptionally important that we gradually refine the lens of our current knowledge on this subject, in particular with the double-blind placebo-controlled method. Unfortunately, this subject is not an area of intensive research at the moment. The methods typically used for diet control may arouse some reservations: nutrition questionnaires, blood tests for n-3 PUFA level (which does not reveal much about nutrition habits in earlier life), and recommendations made in intervention trials that subjects increase their intake of fish or supplement capsules. What is more, the available intervention research typically involves a relatively small sample, and the period of administration of supplements is often shorter than 90 days.

Still, it has not been precisely determined how n-3 PUFAs affect psychological function. There are several possible mechanisms by which EPA and DHA could improve mood in affective disorders. EPA and DHA are intrinsic to the molecular structure of the phospholipids of cell membranes. The fatty acids are crucial to their role in modulating the functioning of proteins in the membrane. PUFAs lend fluidity to cell membranes and have specific functional interactions with membrane enzymes, receptors and other proteins. EPA and DHA can inhibit the protein kinase $C$ signal transduction enzyme complex. They can block 
calcium influx into the cell through the L-type calcium channel, similar to the calcium channel blockers verapamil or nimodipine [24]. However, it should be admitted that current knowledge allows only assuming such mechanisms.

There are also other issues related to the role of $\omega-3$ fatty acids which have yet to be unsolved. First of all, there is not enough data on undesirable side effects of prolonged use of $\omega-3$. Why does a dose of $1-2 \mathrm{~g} / \mathrm{d}$ seem more effective in mood disorders than higher doses? What dose is appropriate in order to limit symptoms of asthma and allergy? What is the optimal proportion of EPA to DHA? And finally, what length of $\omega 3$ PUFAs ingestion is necessary to obtain maximal therapeutic results?

Nonetheless, the significance of $\omega 3$ PUFAs in diet is undeniable, as they have been a natural part of our diet since the rise of humanity. In fact, it is just the past two centuries that 13 PUFAs have been decreased in diets of Western World mainly because of food processing and commercial issues. Many authors call for the need to return to more physiologic n-6:n-3 PUFAs ratio of 1:1 rather than the ratio of about $20: 1$ provided by current western diets.

An optimal prophylactic and therapeutic solutions seem well exemplified by the idea of the Coumbus Concept - a program of innovative nutrient selection, which allows retaining natural and healthy ratios in the most crucial elements. The Columbus Concept assumes that proper function of humans' body and mind is related to the presence of essential nutrients typical for wild food, including $\omega-6 / 3$ fatty acids, antioxidant vitamins and minerals, which supported the upward thrust of human evolution. It is a holistic approach to dietary issues. The Columbus foods include meat, milk, eggs, oil and bread, all rich in n-3 PUFAs with optimal ratios of nutrients, similar to wild foods, consumed about 160 years ago from now.

Hitherto, functional food has not been widely utilized in research on dietary influence on asthma, atopic, and mood disorders. We find it exceptionally crucial to thorough examine the role of essential nutrients in human body and mind, since the optimally balanced diet is likely to become an important step in prophylactics and therapy of many diseases, including mood disorders, asthma, and allergy.

\section{REFERENCES}

[1] McIntyre RS, O'Donovan C. The human cost of not achieving full remission in depression. Can J Psychiatry 2004; 49: 10S-16S.

[2] Fleming DM, Crombie DL. Prevalence of asthma and hay fever in England and Wales. Br Med J 1987; 294: 279-83.

[3] Arbes SJ Jr, Gergen PJ, Elliott L, Zeldin DC. Prevalences of positive skin test responses to 10 common allergens in the US population: results from the third National Health and Nutrition Examination Survey. J Allergy Clin Immunol 2005; 116: 377-83.

[4] Berger WE. Overview of allergic rhinitis. Ann Allergy Asthma Immunol 2003; 90: 7-12.

[5] Zielinski T, Sherwood Brown E, Nejtek VA, Khan DA, Moore JJ, Rush AJ. Depression in Asthma: Prevalence and Clinical Implications. J Clin Psychiatry 2000; 2: 5.

[6] Cooper CL, Parry GD, Saul C, Morice AH, Hutchcroft BJ,
Moore J, Esmonde L. Anxiety and panic fear in adults with asthma: prevalence in primary care. BMC Fam Pract 2007; 8: 62 .

[7] Adams RJ, Wilson DH, Taylor AW, Daly A, Tursan d'Espaighnet E, Dal Grande E, Ruffin RE. Psychological factors and asthma quality of life: a population based study. Thorax 2004; 59: 930-5.

[8] Liu L, Coe C, Swenson C, Kelly E, Kiu H, Busse W. School examination enhance airway inflammation to antigen challenge. Am J Respir Crit Care Med 2002; 165: 1062-7.

[9] Goodwin RD, Castro M, Kovacs M. Major depression and allergy: does neuroticism explain the relationship? Psychosom Med 2006; 68: 94-8.

[10] Janson C, De Backer W, Gislason T, Plaschke P, Bjornsson E, Hetta J, Kristbjarnarson H, Vermeire P, Boman G. Increased prevalence of sleep disturbances and daytime sleepiness in subjects with bronchial asthma: a population study of young adults in three European countries. Eur Respir J 1996; 9(10): 2132-8.

[11] Postolache TT, Stiller JW, Herrell R, Goldstein MA, Shreeram SS, Zebrak R, Thrower CM, Volov J, No MJ, Volkov I, Rohan KJ, Redditt J, Parmar M, Mohyuddin F, Olsen C, Moca M, Tonelli LH, Merikangas K, Komarow HD. Tree pollen peaks are associated with increased nonviolent suicide in women. Mol Psychiatry 2005; 10: 232-5.

[12] Yu G, Bjorksten B. Polyunsaturated fatty acids in school children in relation to allergy and serum $\mathrm{IgE}$ levels. Pediatric Allergy and Immunology 2007; 9(3): 133-8.

[13] Duchén K, Casas R, Fagerås-Böttcher M, Yu G, Björkstén B. Human milk polyunsaturated long-chain fatty acids and secretory immunoglobulin $\mathrm{A}$ antibodies and early childhood allergy. Pediatr Allergy Immunol 2008; 11(1): 29-39.

[14] Olsen SF, Østerdal ML, Salvig JD, Mortensen LM, Rytter D, Secher NJ, Henriksen TB. Fish oil intake compared with olive oil intake in late pregnancy and asthma in the offspring: 16 y of registry-based follow-up from a randomized controlled trial. Am J Clin Nutr 2008; 88 (1): 167-75.

[15] Broughton KS, Johnson CS, Pace BK, Liebman M, Kleppinger KM. Reduced asthma symptoms with n-3 fatty acid ingestion are related to 5 -series leukotriene production. Am J Clin Nutr 1997: 65: 1011-7.

[16] Hibbeln JR. Fish consumption and major depression. Lancet 1998; 351: 1213

[17] Behan PO, Behan WMH, Horrobin D. Effect of high doses of essential fatty acids on the postviral fatigue syndrome. Acta Neurol Scand 1990; 82: 209-16.

[18] Stoll AL, Severus WE, Freeman MP, Rueter S, Zboyan HA, Diamond E, Cress KK, Marangell LB. Omega-3 fatty acids in bipolar disorder: a preliminary double-blind, placebo-controlled trial. Arch Gen Psychiatry 1999; 56: 407-12.

[19] Su K-P, Huang S-Y, Chiu C-C, Shen WW. Omega-3 fatty acids in major depressive disorder: a preliminary doubleblind placebo-controlled trial. Eur Neuropsychopharmacol 2003; 13: 267-71.

[20] Hallahan B, Hibbeln JR, Davis JM, Garland MR. Omega3 fatty acid supplementation in patients with recurrent self-harm: Single-centre double-blind randomised controlled trial. Br J Psychiatry 2007; 190: 118-22.

[21] Fontani G, Corradeschi F, Felici A, Alfatti F, Migliorini S, Lodi L. Cognitive and physiological effects of omega-3 polyunsaturated fatty acid supplementation in healthy subjects. Eur J Clin Invest 2005; 35: 691-9.

[22] Appleton KM, Hayward RC, Gunnell D, Peters TJ, Rogers PJ, Kessler D, Ness AR. Effects of n-3 long-chain polyunsaturated fatty acids on depressed mood: systematic review of published trials. Am J Clin Nutr 2007; 85(6): 1665-6. 
[23] Werneke U, Turner T, Priebe S. Complementary medicines in psychiatry: review of effectiveness and safety. $\mathrm{Br}$ J Psychiatry 2006; 188: 109-201.

[24] Kidd PM. Bipolar disorder as cell membrane dysfunction. Progress toward integrative management. Altern Med Rev 2004; 9(2): 107-35.
Address for correspondence:

Agnieszka Wilczynska-Kwiatek, PhD

Institute of Psychology

University of Silesia

Grazynskiego 53 St.

40-126 Katowice

Poland

Phone: +48601780453

E-mail: Agnieszkawk@hotmail.com agnieszka.wilczynska-kwiatek@us.edu.pl 